\title{
The behaviour of six electrode arrays used in electrical resistivity imaging to detect underground cavities: a numerical study
}

Kamel Hebbache ( $\nabla$ hebbache_kamel@yahoo.com )

University of Biskra https://orcid.org/0000-0002-4409-3689

Djamel Boubaya

University of Tebessa: Universite de Tebessa

\section{Research Article}

Keywords: 2D Electrical resistivity imaging, Electrode array, Cavity, Inversion, L1 norm, Synthetic model

Posted Date: May 3rd, 2021

DOI: https://doi.org/10.21203/rs.3.rs-392621/v1

License: (c) (1) This work is licensed under a Creative Commons Attribution 4.0 International License.

Read Full License 


\section{Abstract}

The aim of this work is to evaluate the efficiency of six electrode arrays used in electrical resistivity imaging. Pole-Pole (PP), Pole-Dipole (PD), Wenner-Alpha (WA), Wenner-Schlumberger (WS), Dipole-Dipole (DD) and multiple Gradient (MG) electrode arrays have been selected to detect underground cavities at shallow depth. Numerical simulation has been made for three synthetic models that have been generated using Res 2 dmod program. Each model represents three cavities with $2 \mathrm{~m}$ diameter, spaced $6 \mathrm{~m}$ from each other and located at a depth of $1.5 \mathrm{~m}$ from the surface of the ground: 1) air-filled cavity, 2) half-watered cavity and 3) full-watered cavity. The background resistivity of each model was chosen equal to 10,50 and $250 \Omega \mathrm{m}$ respectively. The resistivity of the air and water were set at $10^{6} \Omega \mathrm{m}$ and $1 \Omega \mathrm{m}$ respectively. The results show that the PD, MG, PP and WS arrays gave good resolutions and clear images, and are less contaminated by noise. The DD array is very sensitive to noise and for this reason, it gave less accurate results for the first and the second synthetic models. An exception is the third synthetic model, where a good resolution model was obtained. This means, that the DD is more efficient in mapping cavities when the background environment is moderately resistive. The shapes of resistive air-filled cavities were found more clearly than those of conductive watered cavities, for the latter, however, the true resistivity values were better estimated than for the air-filled cavities. From the results of the analysis of the inverted synthetic models, the PD, MG, PP and WS arrays show the best results among the other used electrical arrays.

\section{Introduction}

Detection and delineation of subsurface voids and cavities using near-surface geophysical techniques has gained wide interests over the past few decades. Geophysical techniques aim to identify the feature geometries and to characterize the variations of the physical parameters of underground formations. Techniques used include seismic, electrical resistivity, ground penetrating radar and others that can be used to detect the presence of voids and cavities in subsurface. All of them are based on the assumption by contrasts in the physical properties between the cavities and the surrounding formation. These methods can be used to give information about the subsoil properties, such as thickness of layers, fracture and saturation zones, etc...

The electrical resistivity method is a popular geophysical exploration approach which has been used extensively for natural resources prospecting on a global perspective. It involves determination of the subsurface distribution of electrical resistivity by taking measurements of the potential difference on the ground surface. Electrical resistivity imaging or tomography is a straightforward extension of the classical resistivity method. Electrical resistivity is widely used geophysical method to solve the problems encountered in engineering, environmental protection, resources exploration and hydrogeology. The method is frequently applied to obtain 2D and 3D high resolution images (Griffiths and Barker 1993; Dahlin 1996), in geological investigations including the discovery of cavities (Martínez-Pagán et al. 2013, among other). Resistivity imaging is a useful tool in environmental studies (Šumanovac 2006, among other). It has been tested in geotechnical contexts to assess the relationship between the electrical 
resistivity and basic geotechnical parameters, and the influence of soil moisture content and grain size characteristics (Long et al. 2012). It is also used to image the buried objects such as voids and cavities under various geological conditions (Reynolds 1997; Van Schoor 2002; Abu-Shariah 2009; Martinez et al. 2009). As well as for the estimation of risk of subsidence or collapse of a sinkhole, presenting the topic of several studies (Van Schoor 2002; El-Qady et al. 2005; Fehdi et al. 2011) and many other disciplines can benefit from this measurement technique (see for example Telford et al. 1990; Reynolds 1997).

During the last three decades, an advent of fast computing technologies permitted a broad use of electrical resistivity imaging (Loke and Barker 1996; Dahlin 2001). There have been great improvements in data acquisition systems and progress in resistivity inversion software, so that resistivity imaging has become an increasingly attractive exploration technique (Dahlin and Zhou 2004). Many researchers around the world have demonstrated the possibility to use a large number of measured data by applying of 2D imaging for reconstructing an accurate resistivity image of the subsurface (Sasaki 1994; Loke and Barker 1995; Penz 2012).

Dahlin and Loke, 1997 assessed the effect on 2D resistivity surveying of 3D variation in the resistivity structures and found that the 3D effects in 2D surveying is much less than 2D effects in 1D surveying, i.e. the step from 1D to 2D techniques is more important than the step from 2D to 3D techniques.

The penetration depth in 2D Electrical Resistivity Imaging depends on the resistivity of the medium but can generally be improved by choosing the appropriate array using 2D forward modeling.

The main objective of this study is to examine the effectiveness and applicability of $2 \mathrm{D}$ resistivity imaging technique to detect underground cavities using numerical simulations in conductive and resistive environment background using six different electrode arrays and to evaluate the suitability of their behaviour and resolution. The use of 2D forward modelling will undoubtedly help us to better predict resistivity anomalies associates with underground cavities and to avoid pitfalls (distortion of anomalies) during interpretation by choosing the best electrode arrays.

\section{Electrode configurations}

The value of the observed resistivity depends upon the geometry of the electrode array. Many electrode arrays are used in resistivity method. Each type of array has its own characteristics.

The PP is not used as the WA, DD and WS electrode arrays. This electrode arrangement is defined as the deepest depth of investigation (DOI) and largest horizontal coverage (Loke 2015). However, PP is characterized by its lowest resolution in the inversion model (Dahlin and Loke 1997). The PD (forward and reverse) also has relatively good horizontal coverage and higher signal strength compared with the DD array. The PD is an effective array for detecting vertical structures with a high depth range. The WA array was popular in the early years of electrical imaging due to the straight forward implementation (Dahlin 1996). WA is relatively insensitive to lateral changes in the subsurface resistivity, but it is good for detecting horizontal structures. It provides a reasonable horizontal resolution (Sasaki 1992). The array 
has a moderate depth of investigation (Loke 2000), and generally strong signal strength which is inversely proportional to the geometric factor (Ebraheem et al. 2014). One disadvantage of WA is the relatively poor horizontal coverage with increased electrode spacing.

The WS array is slightly better in terms of horizontal coverage than WA. In comparison with earlier array, the median depth of investigation of WS is deeper. It is a good compromise between WA and DD arrays. The signal strength of WS is higher than DD and weaker than WA array. The DD array is good for mapping vertical structures, such as dikes, fractures and cavities. The DD is more suitable array for resolving complex structures when the instrumental accuracy is high (Sasaki 1992). In general, the median depth of investigation of DD is larger except for the smallest $n$-factors, but also has better horizontal data coverage than WA. The DD has a very low signal strength for large separation factor $(n)$ values. The sensitivity pattern of the DD array depends on the factor $(n)$.

The multiple gradient array (MG) is non-conventional relative to the others. This array is well-suited for multi-channel resistivity meter systems, and it has also good resolution and signal-to-noise ratios (Dahlin and Zhou 2006). The results of this array are comparable to those obtained by the DD and PD arrays (Dahlin and Zhou 2004). It offers a lower sensitivity to noise, which may be a major advantage in real time data acquisition. The sensitivity of $M G$ is very similar to the PD, and it provides very stable field data acquisition. More detailed information of the MG array can be found in (Dahlin and Zhou 2004, 2006).

Among the hundreds of different geoelectric arrays that exist (Szalai and Szarka, 2008a), six arrays are used in this work. The six arrays are: PP, PD, WA, WS and MG. The large number of arrays that exist makes the choice of the appropriate one for surveying very difficult. It is therefore necessary to choose which of the many existing electrode arrays will best respond to changes in contrasting resistivity below the ground surface. On this subject, several studies have been realized in resistivity imaging, in order to compare the results obtained from different electrode arrays (Tamassart (2013); Ogunmola et al.2017). Seaton and Burbey (2002) compared the results from field measurements made using four electrode configurations WA, WS, DD and PP. They concluded that the DD array has the highest resolution and sensitivity to geologic detail and greater depth of investigation than WA and WS, but it has low signal to noise ratio. Dahlin and Zhou (2004) presented a comparison study between 10 electrode arrays, over five synthetic models. They concluded that the following arrays: PD, DD, WS and MG are recommended for 2D resistivity imaging. They summarize the advantage and disadvantage of each electrode array concerning the anomaly effects, signal-to-noise ratios, and imaging capabilities. Reiser et al. (2010) examined the imaging resolution of four electrode arrays for mapping of fracture zones in bedrock (PD, WA, DD and $M G$ ). The best results were obtained with $M G$ and $D D$ arrays, especially for mapping fracture zones with various width and depth. The MG array gives accurate results for mapping dipping structures and different contrast, whereas the good array for illustrating horizontal layers is WA. DD and PD electrode arrays give the most accurate results in the presence of a low resistivity top layer.

\section{Numerical Modeling of Synthetic Data}


The detection of structures and targets below the ground surface using resistivity imaging needs a priori information of the investigated area. The approach used in this study consists of : 1) constructing a theoretical synthetic structure based on geometric models, 2) calculating the apparent resistivity by forward modeling, 3 ) the inversion of generated data obtained by modeling and 4) evaluating the quality of the inverted data.

In this numerical study, we have generated three different synthetic models. Each model represents three cavities with $2 \mathrm{~m}$ diameter, spaced $6 \mathrm{~m}$ from each other and located at a depth of $1.5 \mathrm{~m}$ from the ground surface. The model is set in a way that the center of the fist cavity is located $5 \mathrm{~m}$ from the first electrode position while the second and the third are at 11 and $17 \mathrm{~m}$ respectively. The first model consists of an air-filled, a half-watered and a full-watered cavities, all with a $10 \Omega \mathrm{m}$ resistivity background. The second model is similar to the first but with a $50 \Omega \mathrm{m}$ resistivity background. Similarly, the third and last model has a background resistivity of $250 \Omega \mathrm{m}$.

The first air-filled cavity has been given a resistivity value of $10^{6} \Omega \mathrm{m}$ (magenta). The second half-watered cavity has a resistivity of $1 \Omega \mathrm{m}$ corresponding to salty water (magenta/deep blue). The third cavity which is totally filled with water (water-filled) has also been given a salty water resistivity of $1 \Omega \mathrm{m}$ (deep blue).

The forward modeling is a very useful approach to generate the resistivity response over known subsurface structures. It gives synthetic data that are equivalent to field measurements. In all simulations, the electrical profiles have 48 surface electrodes with $0.5 \mathrm{~m}$ electrode spacing, giving a total profile length of $23.5 \mathrm{~m}$. The forward modeling of synthetic data was carried out using Res2dmod software, version 3.02.06. This forward modeling software calculates the apparent resistivity pseudo-section over synthetic models using the finite element (FE) or the Finite difference (FD) approaches. In the finite difference (FD) approach, the 2D model divides the subsurface into a number of blocks (cells) using a rectangular mesh. Then, the FD method determines the potential distribution at the nodes of the rectangular mesh in both, horizontal and vertical directions. The potential distribution was converted into apparent resistivity values. An ambient random noise distribution of $5 \%$ was added to the synthetic data as voltage dependent to simulate more realistic field conditions. Figure. 2 shows the obtained pseudo-sections of the first synthetic model. It can be seen that, the DD is more contaminated by noise compared to the other arrays (Fig. 2e). The PD, PP, WS and MG are less contaminated by noise, while the WA is insensitive to noise, probably due to its high signal strength (Fig. 2c).

\section{Inverse modeling}

The apparent resistivity data of the three models were inverted using Res2dinv (version 3.71.116). This program uses the finite-difference or finite-element method to calculate the models of $2 \mathrm{D}$ forward response. Res2dinv generates an inverted resistivity-depth image for the profile line based on the leastsquares method (deGroot-Hedlin and Constable 1990; Sasaki 1992; Loke and Barker 1996; Loke et al. 2003). The inversion routines are based on the blocky ( $L_{1}$ norm) (Loke et al. 2003) and smoothnessconstrained least-squares method ( $L_{2}$ norm) optimizations (DeGroot-Hedlin and Constable 1990; Loke 
and Dahlin 2002). The L1 norm minimizes the absolute values of the data misfit in order to give a model. (Loke and Dahlin, 2002) showed that the $L_{1}$ norm is less sensitive to noisy data points but might give a higher apparent resistivity RMS error. On the other hand, the $L_{2}$ norm requires a smooth model that minimises the squares of data misfit. With a larger misfit, L2 norm gives greater importance to data points and this makes it very sensitive to the outliers. Res2dinv uses by default the $\mathrm{L}_{2}$ norm optimization technique. Table 1 summarises the main parameters of inversion that have been used in this study.

\section{Results And Discussion}

The inverted sections obtained from the 2D inversion of the six electrode arrays with $5 \%$ free noise are shown in figures 3,4 and 5 . The inversions were performed applying robust data and model constraints. The robust inversion option was used because cavities in most cases have sharp geologic boundaries. The inverted model should resemble the initial synthetic model (Rønning et al 2014). Deviations are caused by weaknesses in the inversion procedure and/or methodological limitations such as resolution.

Figure $3 a$ to $f$ shows the results of the first synthetic model with background resistivity of $10 \Omega \mathrm{m}$. The RMS (table.1) varies between a minimum of $2 \%$ for WA to $21.5 \%$ for DD. As it is expected the WA, MG, WS and PD were less sensitive to noise, while the DD was more sensitive to noise. On this model, the top of the resistive cavities (air-filled and half-watered) have been well recovered and imaged (station 5 and 11) by PP, PD, WS and MG. The resistive part of the half-watered cavity was not imaged by WA (fg.3.c). It should be noted that, the bottom of the air-filled cavity was not well resolved by the WA, WS and MG arrays. The DD with a RMS of $21.5 \%$ was very contaminated by noise and did not gave clear images for the first synthetic model (fg.3e).

The conductive part of the half-watered cavity (station 11) was not imaged by all arrays. It seems that it is located beyond the detection limit (it is quite deep and small in size).

The top part of the conductive full-watered cavity, located below the station 17, was highlighted by all arrays. However, its diameter is quite large (PP, WA, WS), and its bottom is unresolved. There is a resistive artefact around the top of this cavity for the PD array.

The results of the second synthetic model ( $\mathrm{fg} .4 \mathrm{a}$ to $\mathrm{f}$ ) with background resistivity of $50 \Omega \mathrm{m}$ were similar to the first synthetic model. The DD also, with a RMS of $25.5 \%$ showed resistive artefact above the recovered cavities.

The third synthetic model (fg.5a to f) with a background resistivity of $250 \Omega \mathrm{m}$ made it possible to image the resistive and conductive cavities with all arrays. However, the width and the bottom of the conductive cavity situated below station 17 were inaccurate. DD gave clear images for this third synthetic model (fg.5e) that are similar to PP and PD. This means, that the DD is more efficient in mapping cavities when the background environment is moderately resistive. 
The shapes of resistive cavities were better recovered than those of conductive cavities. The phenomenon is reversed with regard to the obtained resistivity values. This behaviour is explained by the focusing of the current lines towards the conductive targets and the fact that they bypass the resistive zones (Penz 2012). Considering the results obtained, the ranking of the performance of the six arrays in terms of cavity detection for our particular synthetic models is as follows: PD, MG, PP, WS, WA and DD.

\section{Conclusion}

In this study, we investigated the 2D resistivity imaging capability and efficiency of six electrode arrays in order to detect underground cavities at shallow depth. Air-filled, half-watered and full-watered cavities in different background resistivities have been synthetically modeled. The obtained results from this numerical study show the advantages and limitations of each electrode array. The PD, MG, PP and WS arrays are the most suitable for characterizing underground cavities at shallow depth. They gave good resolution, clear images, and were less contaminated by noise. It was found that the DD is effective in detecting cavity with a moderately resistive background. The shapes of resistive air-filled cavities were found more clearly than those of conductive watered cavities, for the latter, however, the true resistivity values were better estimated than for the air-filled cavities. The performance of the six arrays in terms of cavity detection for our particular synthetic models is as follows: PD, MG, PP, WS, WA and DD.

\section{Declarations}

\section{Acknowledgement}

The authors are very grateful to Dr M.H. Loke (Geotomo Software Penang, Malaysia) for his help during the process of forward modeling using Res2dmod Software. Kamel Hebbache thanks Pr. Torleif Dahlin (Lund University, Sweden) for his support and assistance during the processing and inversion of synthetic resistivity data.

\section{References}

Abu-Shariah MII (2009) Determination of cave geometry by using a geoelectrical resistivity inverse model. Eng Geol 105:239-244.

Bayrak M, Şenel, L (2012) Two-dimensional resistivity imaging in the Kestelek boron area by VLF and DC resistivity methods. J Appl Geophys 82:1-10

Dahlin, T (1993) On the Automation of 2D Resistivity Surveying for Engineering and Environmental Applications. PhD Thesis Department of Engineering Geology, Lund University, p187.

Dahlin, $T$ (1996) 2D resistivity surveying for environmental and engineering applications. First Break $14: 275-283$. 
Dahlin T (2001) The development of DC resistivity imaging techniques. Comput Geosci 27:1019-1029.

Dahlin T, Loke MH (1997) Quasi-3D resistivity imaging-mapping of three-dimensional structures using two-dimensional DC resistivity techniques. Proceedings of the 3rd Meeting of the Environmental Engineering Geophysics Society, pp 143-146.

Dahlin T, Zhou B (2004) A numerical comparison of 2D resistivity imaging with 10 electrode arrays. Geophys Prospect 52:379-398.

Dahlin T, Zhou B (2006) Multiple gradient array measurements for multichannel 2D resistivity imaging. Near Surf Geophys 4:113-123.

DeGroot-Hedlin C, Constable S (1990) Occam's inversion to generate smooth, two-dimensional models from magnetotelluric data. Geophysics 55:1613-1624.

Dey A, Morrison HF (1979) Resistivity modelling for arbitrarily shaped two-dimensional structures. Geophys Prospect 27:106-136.

Ebraheem AM, Al Mulla MM, Sherif MM, Awad O, Akram SF, Al Suweidi NB, Shetty A (2014) Mapping groundwater conditions in different geological environments in the northern area of UAE using 2D earth resistivity imaging survey. Environ Earth Sci 37:1555-1641.

El-Qady G, Hafez M, Abdalla MA, Ushijima K (2005) Imaging subsurface cavities using geoelectric tomography and ground-penetrating radar. J Cave Karst Stud 67:174-181

Fehdi C, Baali F, Boubaya D, Rouabhia A (2011) Detection of sinkholes using 2D electrical resistivity imaging in the Cheria Basin (north-east of Algeria). Arab J Geosci 4:181-187.

Griffiths DH, Barker RD (1993) Two-dimensional resistivity imaging and modeling in areas of complex geology. J Appl Geophys 29:211-226.

Li Y, Oldenburg DW (1992) Approximate inverse mappings in DC resistivity problems. Geophys J Int 109:343-362

Loke MH (2000) Electrical imaging surveys for environmental and engineering studies. Technical note of Geotomo Software. Available on line at http://www.geoelectrical.com.

Loke MH (2015) Tutorial: 2-D and 3D electrical imaging surveys : http://www.geoelectrical.com Loke MH, Barker RD (1995) Least-square deconvolution of apparent resistivity pseudo-sections. Geophysics 60(6):499-523.

Loke MH, Barker RD (1996) Rapid least-squares inversion of apparent resistivity pseudosections by a quasi-Newton method. Geophys Prospect 44:131-152 
Loke MH, Acworth I, Dahlin T (2003) A comparison of smooth and blocky inversion methods in 2D electrical imaging surveys. Explor Geophys 34:182-187.

Loke M, Dahlin T (2002) A comparison of the Gauss-Newton and quasi-Newton methods in resistivity imaging inversion. J Appl Geophys 49:149-162.

Long M, Donohue S, L'Heureux JS, Solberg IL, Rønning JS, Limacher R, O'Connor P, Sauvin G, Rømoen M, Lecomte I (2012) Relationship between electrical resistivity and basic geotechnical parameters for marine clays. Can Geotech J 49(10):1158-1168.

Martinez J, Benavente J, García-Aróstegui JL, Hidalgo MC, Rey J (2009) Contribution of electrical resistivity tomography to the study of detrital aquifers affected by seawater intrusion-extrusion effects: the river Vélez delta (Vélez-Málaga, southern Spain). Eng Geol 108:161-198.

Martínez-Pagán P, Gómez-Ortiz D, Martín-Crespo T, Manteca J, Rosique M (2013) The electrical resistivity tomography method in the detection of shallow mining cavities. A case study on the Victoria Cave, Cartagena (SE Spain). Eng Geol 156:1-10

Nguyen F, Garambois S, Jongmans D, Pirard E, Loke MH (2005) Image processing of 2D resistivity data for imaging faults. J Appl Geophys 57(4):260-277

Ogunmola F J, Adetola SO and Olajumoke A D (2017) Numerical modeling of geologic structures using $2 d$ resistivity imaging with seven electrode arrays. FUW Trends in Science \& Technology Journal Vol. 2 No.1A: 126-136

Penz S (2012) Modélisation et inversion de données électriques en courant continu : vers une prise en compte efficace de la topographie. Phd Thesis. Ecole Nationale Supérieure des Mines de Paris.

Reiser F, Dalsegg E, Dahlin T, Ganerød GV, Rønning JS, Lindstrøm M (2010) Resistivity modelling of fracture zones and horizontal layers in bedrock. Report, NGU (Norwegian Geological Survey)

Reynolds, J,M (1997) An introduction to applied and environmental geophysics. Wiley, Chichester.

Rønning J S, Ganerød G V,DalseggE, Reiser, F (2014) Resistivity mapping as a tool for identification and characterisation of weakness zones in crystalline bedrock: definition and testing of an interpretational model. Bulletin of Engineering Geology and the Environment. vol. 73 (4)

Sasaki ,Y (1992). Resolution of resistivity tomography inferred from numerical simulation. Geophysical Prospecting 40:453-464.

Sasaki, Y (1994) 3-D resistivity inversion using the finite-element method. Geophysics 59:1839-1848 Seaton WJ, Burbey TJ (2002) Evaluation of two-dimensional resistivity methods in a fractured crystallinerock terrane. J Appl Geophys 51:21-41. 
Storz H, Storz W, Jacobs F (2000) Electrical resistivity tomography to investigate geological structures of the earth's upper crust. Geophys Prospect 48:455-471.

Stummer P, Maurer H, Green AG (2004) Experimental design: Electrical resistivity data sets that provide optimum subsurface information. Geophysics 69:120-139.

Šumanovac F (2006). Mapping of thin sandy aquifers by using high resolution reflection seismics and 2D electrical tomography. J Appl Geophys 58:144-157.

Szalai, S et Szarka, L (2008a) Parameter sensitivity maps of surface geoelectric arrays I : Linear arrays. Acta. Geod. et Geoph. Hung., 43(4), 419-437.

Tamssar, A H (2013) An evaluation of the suitability of different electrode arrays for geohydrological studies in Karoo rocks using electrical resistivity tomography. Ph.D. Thesis, university of Free State, faculty of natural and agricultural science, south Africa.183p

Telford W,M, Gildart LP, Sheriff RE (1990) Applied geophysics, 2nd edn. Cambridge University Press, Cambridge.

Van Schoor M (2002) Detection of sinkholes using 2D electrical resistivity imaging. J Appl Geophys 50:393-399.

Zhou B, Dahlin T (2003) Properties and effects of measurement errors on 2D resistivity imaging surveying. Near Surf Geophys 1(3):105-117.

\section{Tables}

Table 1 Density of data points and RMS error of each electrode array

\begin{tabular}{|c|c|c|c|c|}
\hline Electrode array & \multirow{2}{*}{$\begin{array}{c}\text { Data } \\
\text { coverage }\end{array}$} & \multicolumn{3}{|c|}{ RMS error of the inverted 2D resistivity sections } \\
& & $1^{\text {st }}$ & \\
\cline { 3 - 5 } & & 4.5 & 1.8 & $3^{\text {rd }}$ Model \\
\hline Pole-Pole & 632 & 7 & 1.9 & 1.4 \\
Pole-Dipole (For and & 1546 & 2 & 2 & 1.4 \\
Rev) & 360 & 4.6 & 1.9 & 1.8 \\
Wenner-Alpha & 1060 & 21.5 & 25.5 & 1.8 \\
Wenner-Schlumberger & 1260 & 2.3 & 1.5 & 3.8 \\
Dipole-Dipole & 906 & & & 1.6 \\
Gradient & & & & \\
\hline
\end{tabular}


Table 2 Inversion parameters

\begin{tabular}{|l|l|}
\hline \multicolumn{1}{|c|}{ Inversion Parameters } & \multicolumn{1}{|c|}{ Values } \\
\hline Initial damping factor & 0.15 \\
Minimum damping factor & 0.03 \\
RMS convergence limit & $2 \%$ \\
& 1.1 \\
Increase of the damping factor with depth & $3-10$ \\
Number of iterations & 2 \\
Vertical to horizontal flatness filter ratio & 4 \\
& Gauss-Newton \\
Number of nodes between adjacent electrodes & Robust/Smooth \\
& 0.05 \\
Type of Jacobian matrix calculation & Robust/Smooth \\
Data constraint & 0.005 \\
Cutoff factor for robust data constraint & Yes \\
Model constraint & Slight \\
Cutoff factor for robust model constraint & Fine \\
Extended model & Yes \\
Reduce the effect of side blocks & Half-width \\
Type of mesh & cells \\
Blocks have the same width & \\
Model refinement & \\
& \\
\hline
\end{tabular}

\section{Figures}




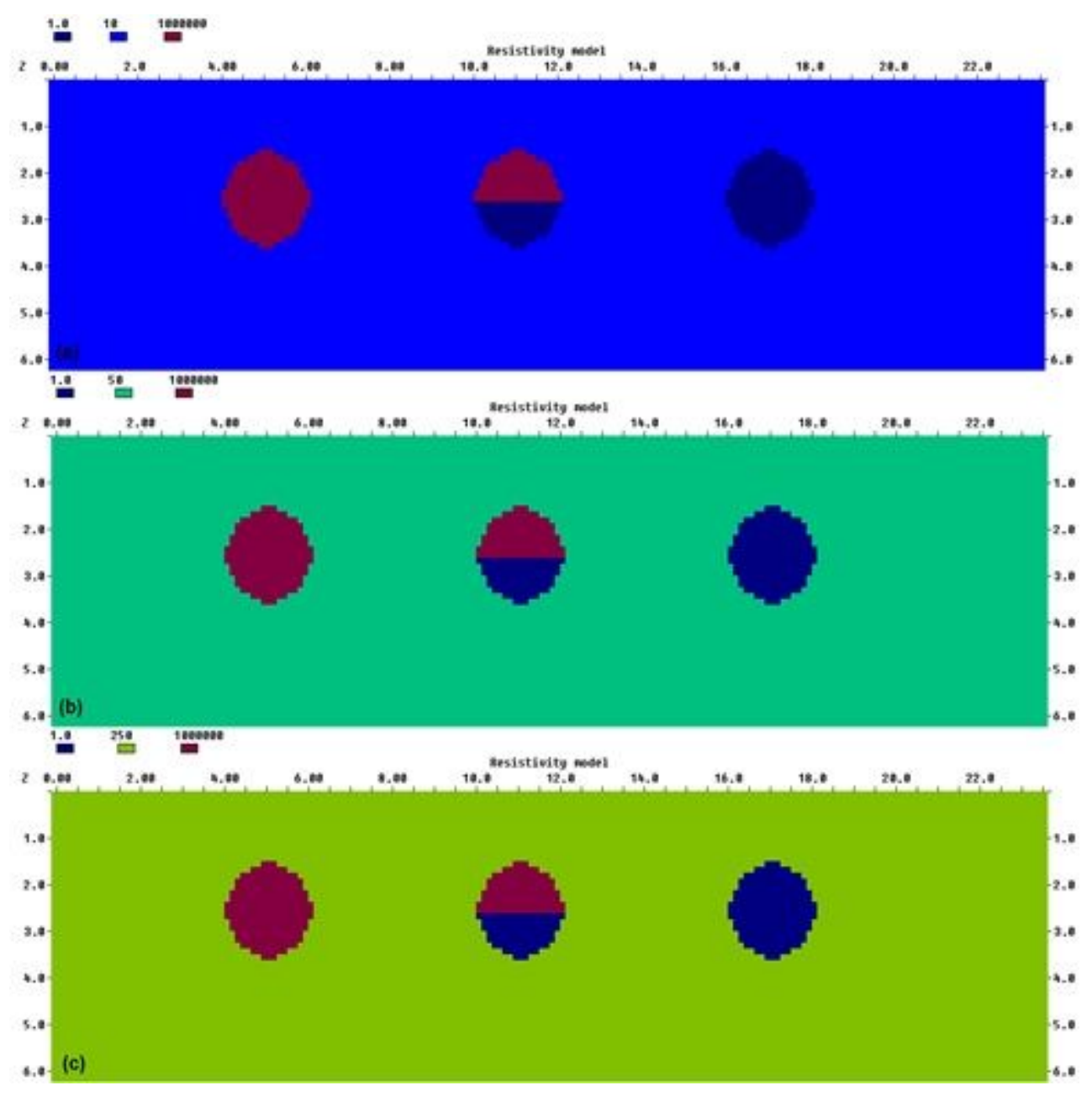

\section{Figure 1}

Resistivity models. a First model, b Second model and c Third model 


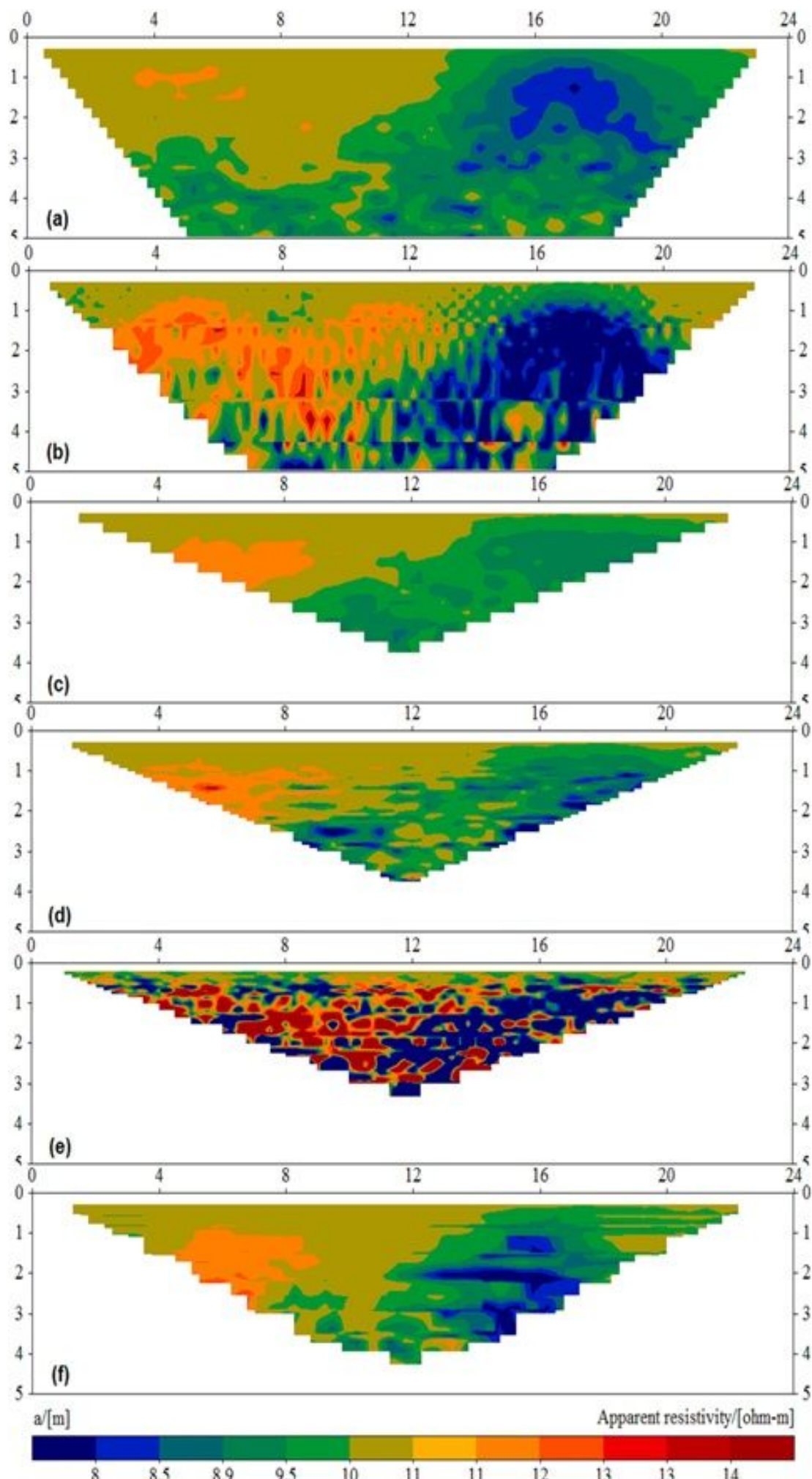

Figure 2

Pseudo-sections of noise-contaminated voltage dependent generated of the first model: a Pole-pole, $b$ pole-dipole, c Wenner-Alpha, $d$ Wenner-Schlumberger, e dipole-dipole and $f$ multiple gradient array 

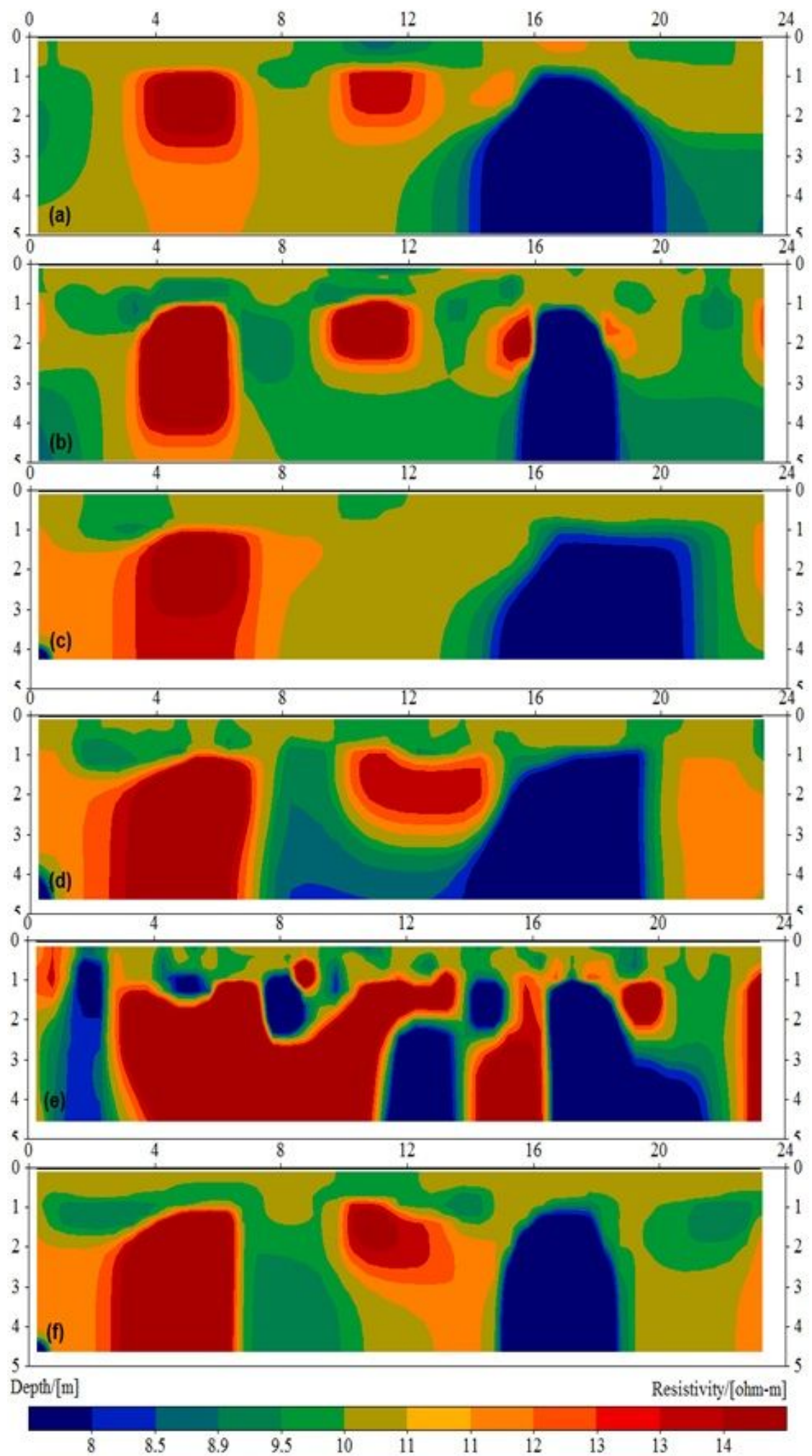

Figure 3

Inverted 2D resistivity sections of the first synthetic model: a Pole-pole, b pole-dipole, c Wenner-Alpha, d Wenner-Schlumberger, e dipole-dipole and $\mathrm{f}$ multiple gradient array 

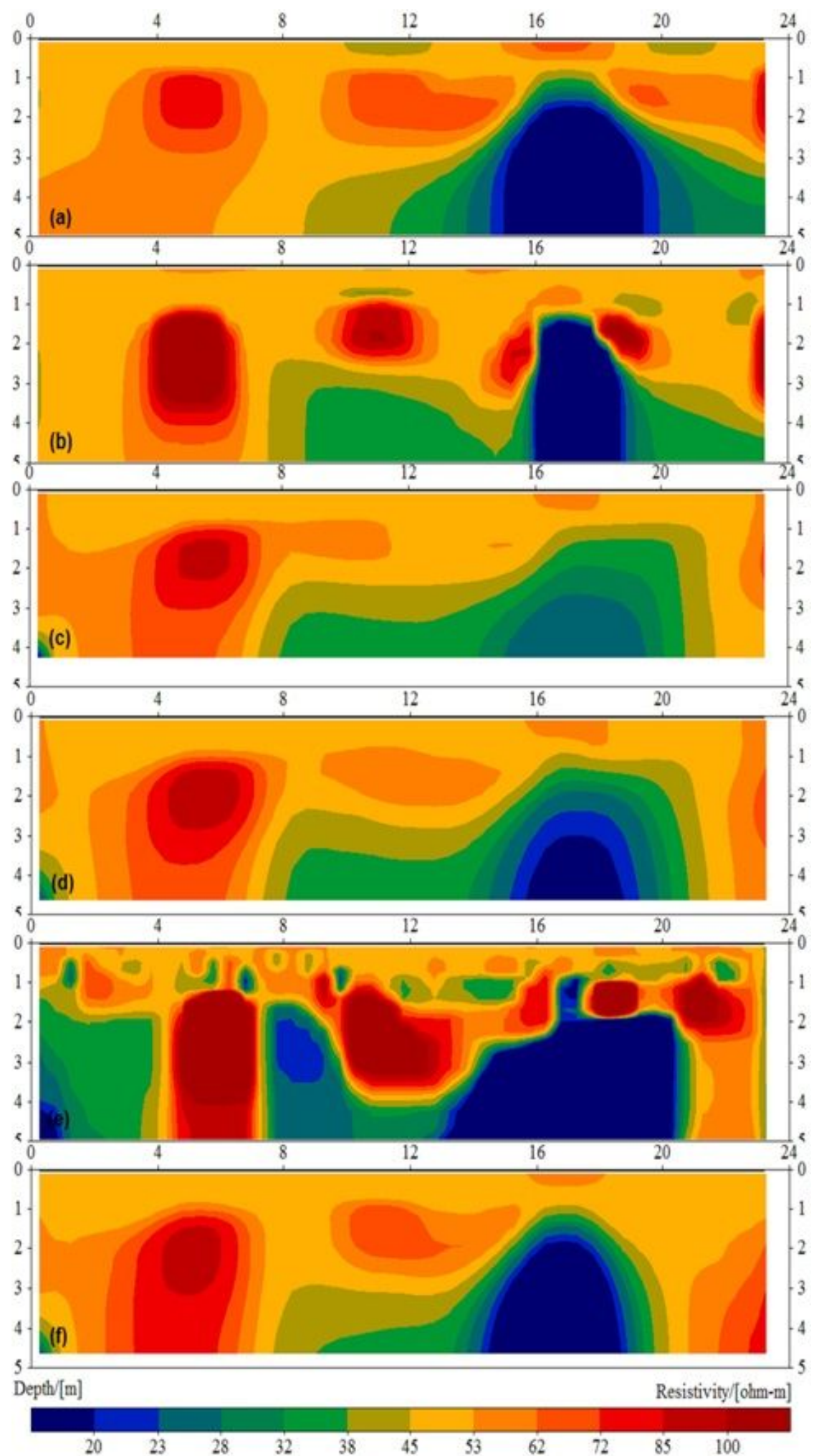

Figure 4

Inverted 2D resistivity sections of the second synthetic model: a Pole-pole, b pole-dipole, c Wenner- Alpha, $\mathrm{d}$ Wenner-Schlumberger, e dipole-dipole and $\mathrm{f}$ multiple gradient array 


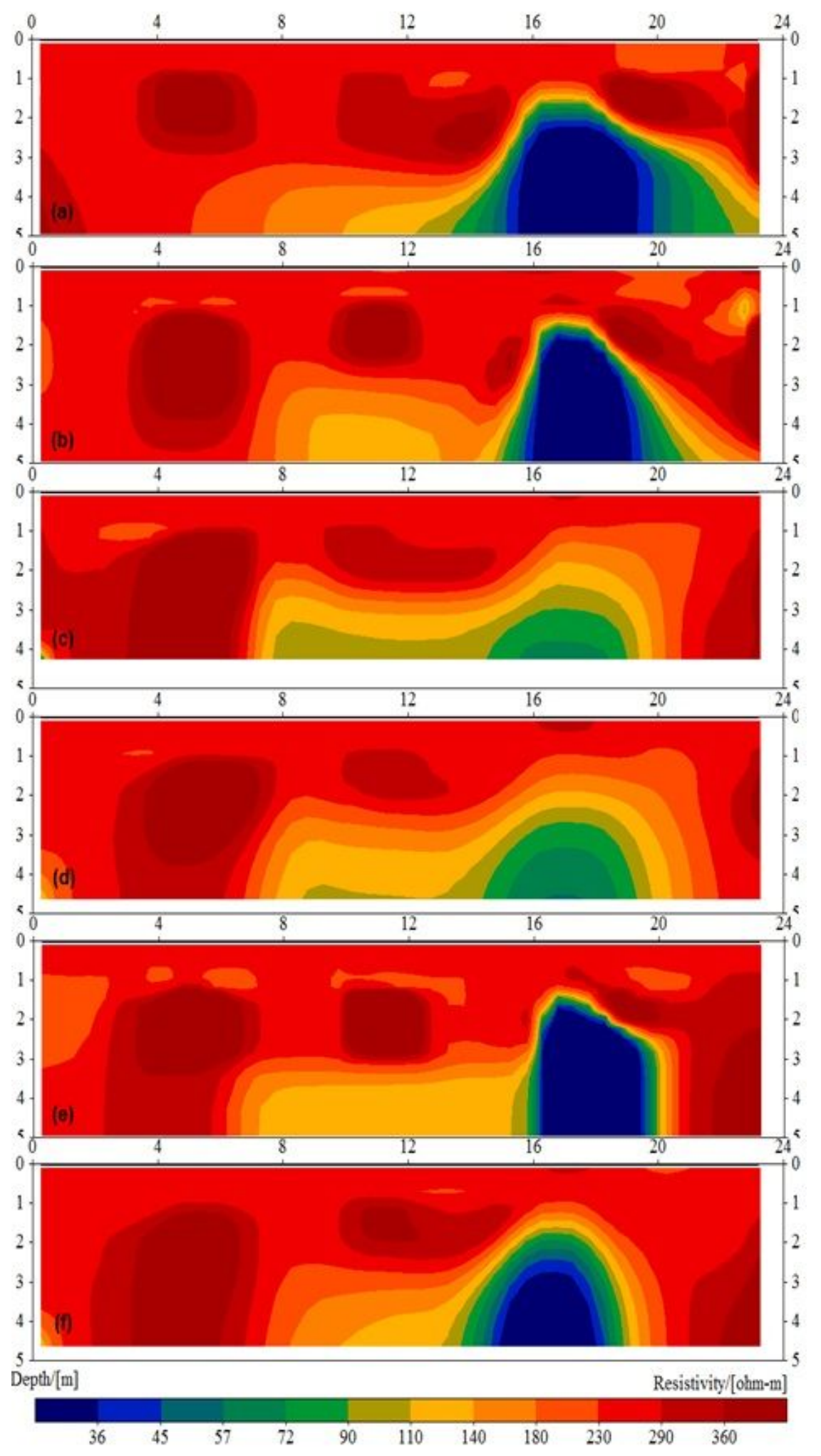

Figure 5

Inverted 2D resistivity sections of the third synthetic model: a Pole-pole, b pole-dipole, c Wenner- Alpha, d Wenner-Schlumberger, e dipole-dipole and $\mathrm{f}$ multiple gradient array 